Meta

Journal des traducteurs

Translators' Journal

\title{
La traduction à quatre roues
}

\section{Robert Larose}

Volume 40, numéro 3, septembre 1995

La traduction, qu'est-ce à dire? Phénoménologies de la traduction

URI : https://id.erudit.org/iderudit/003408ar

DOI : https://doi.org/10.7202/003408ar

Aller au sommaire du numéro

Éditeur(s)

Les Presses de l'Université de Montréal

ISSN

0026-0452 (imprimé)

1492-1421 (numérique)

Découvrir la revue

Citer cet article

Larose, R. (1995). La traduction à quatre roues. Meta, 40(3), 406-408. https://doi.org/10.7202/003408ar

\section{Résumé de l'article}

Translation has been associated to traitors, mirors, unfaithful women... and, more recently, to a large-scale means of mass transportation: the automobile. But is translation a four-wheel drive? How does one go about approching his/her object of inquiry, whether it be an automobile or translation? What are the access roads, the bumps and dead ends? Shouldn't researchers take a driver's seat approach to the open road? Finally, is translation merely a vehicule for traveling between places, or can it also be a metonymical figure for something else? 


\title{
LA TRADUCTION À QUATRE ROUES
}

ROBERT LAROSF

l'niversité de Momtréal. Montréal. Canada

\begin{abstract}
Translation has been associated to traitors, mirors, unfaithful women... and. more recently, to a large-scale means of mass transportation: the automohile. But is translation a four-nhed drive" How deses one gor about approching his/her object of inquiry, whether it be an automohile or stanslation." What are the acoess roads, the humps and dead ends." Shouldrit researchers take a driver's seat approak he the open road? Finally, is ranslation merely a vehicule for traveling between places. or can it also be a metomymic al figure for sromething else."
\end{abstract}

Au cours des siècles, la traduction a fait l'objet de nombreux clichés épigrammatiques où déferlent les associations avec des manteaux, traîtres, miroirs, pièces de monnaie, revers de tapisserie et femmes infidèles.

Il faut bien l'avouer, les tapisseries ne sont guère monnaie courante aujourd'hui. Quant aux femmes infidèles... étant donné le climat de politic al correctness qui règne par les temps qui courent. mes collègues $m$ 'ont fortement recommandé d'éviter une embardée certaine et de filer une métaphore plus «neutre». ce qui $m$ 'a amené à faire marche arrière et à choisir celle de l'automobile.

Peut-être trouvera-t-on prosaïque de comparer la traduction à quelque chose d'aussi utilitaire que l'automobile. De fait. la démocratisation de cette dernière a banalisé son image. Mais l'histoire de ses "formes» $n$ 'en demeure pas moins la manifestation concrète de courants esthétiques bien réels. qui ont évolué au fil des ans.

Il arrive parfois que les critiques lèvent le nez sur les objets de consommation de masse. Pour s’en convaincre, il suffit de considérer les accusations de dérapage formulées à l'endroit des dirigeants du Musée des beaux-arts de Montréal qui ont eu l'«audace» d'organiser, du 11 mai au 15 octobre 1995. une exposition sur l'automobile intitulée Beauté mobile. exposition qui n'affichait rien du kitsch des salons traditionnels de l'automobile. Cependant. certaines personnes n'ont pu tolérer qu'elle se déroule dans un musée' plutôt qu'au stade olympique ou au palais des congrès, ni qu'elle porte sur un objet utilitaire (mol combien capotant!) qui suppose un travail collectif. Devrait-on croire que seuls les objets inutiles ou de luxe seraient dignes de légitimation artistique de la part des instances de pouvoir de la ahaute" culture ?

Afin de savoir si la traduction a bel et bien quatre roues (outre, bien sûr, la roue de secours) ou encore s'exerce à quatre roues, l'analyste doit s'interroger sur son point de départ. Ainsi, pour être en mesure de répondre à ce que mon collègue Alexis Nouss nomme l'objection principielle (de quoi parle-t-on ?). l'analyste doit jalonner son objet d'étude, c'est-à-dire planter devant ou autour de celui-ci un ou plusieurs jalons-mires bien définis qui lui permettront d'observer la mécanique. le coût de production (l'économie mène le monde, disait Marx, ne nous en déplaise), le rôle social du produit. son esthétique, ses étapes de fabrication ou son uessence». Sans repères conceptuels clairs, le regard risque de se brouiller et les propos, de se diluer en molles généralités. Force est de reconnaître néanmoins que le champ d'investigation. qu'il s'agisse de la traduction ou de l'automobile, est toujours arpenté partiellement. De surcroît, il est improbable qu'on élimine un jour la part de subjectivité de l'analyste, dont les besoins et les valeurs conditionnent son regard. "L'homme, disait Protagoras, est la mesure de toute 
chose.» Or, c'est précisément la somme de ces «regards» sur un objet qui permet d'en cartographier la complexité. tout comme c'est l'ensemble des emplois d'un mot qui en constitue le sens. Utopie fantasmagorique associée au désir d'exhaustivité du chercheur?

La complexité d'appréhension ne doit toutefois pas freiner ce désir. Qu'il s'agisse d'un texte, d'une voiture ou... d'une femme (avec nos excuses contrites aux féministes les plus farouches), ce sont les techniques d'approche qui comptent... et leur diversité représente autant de «voies d'accès» à l'objet, qu'il se nomme automobile ou traduction :

- métaphysique : qu'est-ce que l'automobile ? (question d'une simplicité déroutante) / ' 'esprit (noûs, en grec) d' un texte peut-il voyager d' une langue d une autre?

- éthique : que signifie conduite dangereuse ? / d partir de quand une traduction dérape-t-elle?

- didactique : quel équipement est indispensable à l'enseignement de la mécanique automobile? / que peut-on bien vouloir entendre par enseignement de la traduction "générale"?

- descriptive : quelles sont les caractéristiques de la Renault Clio ? / en quoi consiste l'oralité en traduction théâtrale?

- socio-historique : quels pays construisent des voitures, en quelle quantité, de quels types et depuis quand? / quelles sont les formes prises par la censure victorienne et quels en sont les effets sur la traduction des romans de Zola?

- analytique : quels sont les avantages et les inconvénients du moteur Wankel ?/ quels parametres permettraient d'affirmer que les phonemes sont bel et bien porteurs de sens $d^{\prime}$ une langue d une autre?

- comparée : sur le plan aérodynamique, laquelle parmi la Infiniti Q45, la Lexus LS 400 et la Lincoln Continental présente le meilleur Cx? / que dégage-t-on de la comparaison des versions de Hamles faites par A. Dumas et P. Meurice (1847). A. Gide (1938) et Y. Bonnefoy (1962)?

- esthétique (poétique): a-t-on jamais créé style plus audacieux que celui de l'Alfa-Romeo B Aerodinamica 1934 conçue par l'écurie d'Enzo Ferrari ? / l'approche poéricienne de H. Meschonnic rend-elle la littérarité de la Bible?

- économico-politique: pourquoi le Québec ne construit-il pas sa propre voiture? / pourquoi n'a-t-il pas de politique de traduction, en particulier pour ce qui est des secteurs culturels?

- industrielle : quelles sont les étapes de fabrication d'une voiture? / pourquoi l'Ordre des traducteurs el interprètes agréés du Québec (OTIAQ) n'a-t-il pas encore souscrit d la norme ISO 900)4-2?

- pragmatique : le produit livré correspond-il aux exigences énoncées dans le cahier des charges? / le virage clientéliste permet-il de produire des traductions plus efficaces?

- psychologique : que se passe-t-il dans l'esprit du concepteur au moment de dessiner une fourgonnette? I ne rombe-t-on pas toujours en panne quand le moment est venu d'expliquer ce qui se passe dans le cerveau du traducteur?'

- psychanalytique : est-il normal de conduire à 200 à l'heure sur les ChampsÉlysées ? I" où vient lo "passion" de traduire, ce moteur de lart?"

- déconstructiviste : quelles sont les pièces essentielles d'une voiture ?/ est-ce qu'il y a vraiment un texte au départ?

- postmoderne : une Chevrolet Firefly 1995 ne vaut-elle pas une Talbot-Lago T150 SS 1938? / une traduction. même jugée bonne par la critique. n'est-elle pas toujours considérée comme mausaise, de par sa nature de traduction?

- etc. 

allongée ?

Que révèlent les rapprochements ci-dessus, dont la liste pourrait être indéfiniment

$1 \%$ Qu'ils sont nombreux et «éclatés». Il est vrai que l'anarchie est souvent une condition indispensable au développement d'un champ du savoir, mais l'instabilité permanente risque au contraire de ne pas lui convenir, surtout si elle aspire au statut de discipline universitaire reconnue. Aussi faudra-t-il sans doute établir pour la traduction un arbre des domaines (pour mieux voir la forêt ?) comparable à ce qui existe déjà en terminologie automobile.

$2 \%$ Qu'il n'existe pas de sauts-de-mouton intradisciplinaires, c'est-à-dire entre les divers thèmes de rapprochements. Autrement dit. sans schéma intégré, sans dispositif de cerveau-direction qui les rattacherait les unes aux autres, à la façon d'une toile d'araignée, les démarches demeureront atomisées et leur ensemble restera statique et stratifié.

$3 \%$ Qu'il n'y a pas de ponts interdisciplinaires qui relieraient la traduction aux autres champs du savoir. La traduction $n$ 'est pas seulement transport, elle est aussi rapport avec le monde, fragment d'ensembles eux-mêmes fragmentés, dimension sur laquelle prend appui une autre figure : la métonymie.

$4^{\circ} / \mathrm{Qu}$ 'il n'y a pas non plus de tout-terrain «méta-théorique» à l'aide duquel l'analyste jugerait de la tenue de route de chacune des approches, selon un axe allant des objectifs visés aux fins réalisées. L'analyse multi-critères que nous avons décrite dans "Qualité et efficacité en traduction» (Meta, 39-2, $1994: 367-369)$ en représente d'ailleurs l'outil essentiel. (Dans ce même article, nous avons cité deux extraits, l'un de Halliday, l'autre de Simpkin, dans lesquels les auteurs recourent eux aussi à la métaphore de l'automobile.)

$5 \%$ Que la perception extérieure de l'objet, qui est bien une expérience de l'objet lui-même en phénoménologie. ne suffit pas à en découvrir le moteur. Une double exigence heuristique a été oubliée : aller sous le capot et derrière le volant. On pourra alors espérer comprendre davantage les principes et les propriétés qui régissent le mouvement et, en s'exposant à l'expérience ${ }^{2}$ de la traduction, viser ainsi une meilleure conscience de l'être-en-traduction. Ce déplacement épistémologique débouche sur une ontologie du traducteur qui le place dans le véhicule et au volant de celui-ci.

Enfin. comme la métaphore de l'automobile implique celle du transport - mouvement d'embrayage sur un autre lieu - et celle de la métempsycose - changement d'état, la traduction en vient à revêtir la forme d'une transmigration. Ainsi, ce qui change devient ce qu'il n'est pas, bien que la permanence dans le devenir soit précisément le changement.

\section{Notes}

1. Pourtant. en 1970. le musée des Arts décoratifs de Paris avait organisé une très belle exposition intitulee Bolide design, consacrée aux voitures de course, et. en 1994. le Museum of Modem Art de New York a présenté l'exposition ferrari.

2. Dans ses Méditations cartésténnes, Edmund Husserl écrit : "Je ne pourrai évidemment ni poner ni admettre comme valable aucun jugement. st je ne l'ai puisé dans l'ésidence, c'est-à-dire dans des 'expériences' où les 'choses' et 'faits' en question me sont présents 'eux-mêmes'" (Librairie J. Vrin. Paris, 1966: 11 ). 